\title{
PLASMA DIAGNOSTICS IN VIEW OF ITER
}

\author{
A.J.H. Donné \\ FOM-Institute DIFFER, Dutch Institute for Fundamental Energy Research, Assocation EURATOM-FOM, \\ Partner in the Trilateral Euregio Cluster, P.O. Box 1207, 3430 BE Nieuwegein, The Netherlands, www.differ.nl \\ Tel. +31 306096 960, Fax +31 306031 204, e-mail: a.j.h.donne@differ.nl
}

Also Eindhoven University of Technology, Dept. of Applied Physics, PO Box 513, 5600 MB Eindhoven, The Netherlands

\begin{abstract}
The ITER environment imposes many challenges for the various diagnostic systems. At the one hand diagnostic functionalities are required that go well beyond those at present devices. This is because there is a need to actively control (the profiles of) multiple plasma parameters, implying that measurement systems should be accurate and reliable. At the other hand the application of diagnostics at ITER is strongly hampered by constraints arising from the relatively harsh environmental conditions that give rise to phenomena that are new to the diagnostic designs. The nuclear environment puts stringent demands on the engineering and robustness of diagnostics, while the long pulse lengths require high stability of all systems. This paper will present an overview of the diagnostics for ITER with an additional glance in the further future.
\end{abstract}

\section{INTRODUCTION}

The step to ITER diagnostics is the most substantial challenge ever encountered in the development of diagnostics for high temperature plasmas: ${ }^{1}$

- The measurement requirements on ITER are much more stringent than in present devices. Many diagnostics are incorporated in real-time feedback loops (e.g for the stabilization of magnetohydrodynamic modes, for controlling the current profile, for controlling the presence and properties of internal transport barriers), requiring a high level of reliability and availability.

- The diagnostics have to cope with a much harsher environment than in present devices. Radiation-induced effects strongly restrict the use of insulators and of refractive components close to the plasma. Diagnostics need to be robust, such that they can survive during the complete ITER operational life time, or if that is not possible, such that they only need rather infrequent replacements or maintenance.

- The long ITER pulses put severe constraints on the stability and reliability of the diagnostics.

- Multiple diagnostics have to share a single diagnostic port, which creates many interfaces between different diagnostics which require well organized project teams and a high degree of quality assurance.
In this paper a brief overview will be given of the challenges in the design and manufacturing of the diagnostics for ITER, using Fig. 1 as a guideline. The most comprehensive paper that has been published thus far on ITER diagnostics, ${ }^{1}$ as part of the ITER Physics Basis, contains a wealth of information on all aspects that will be covered in this paper. Instead of repeating that information here, the reader will be in several cases referred to tables in that paper.

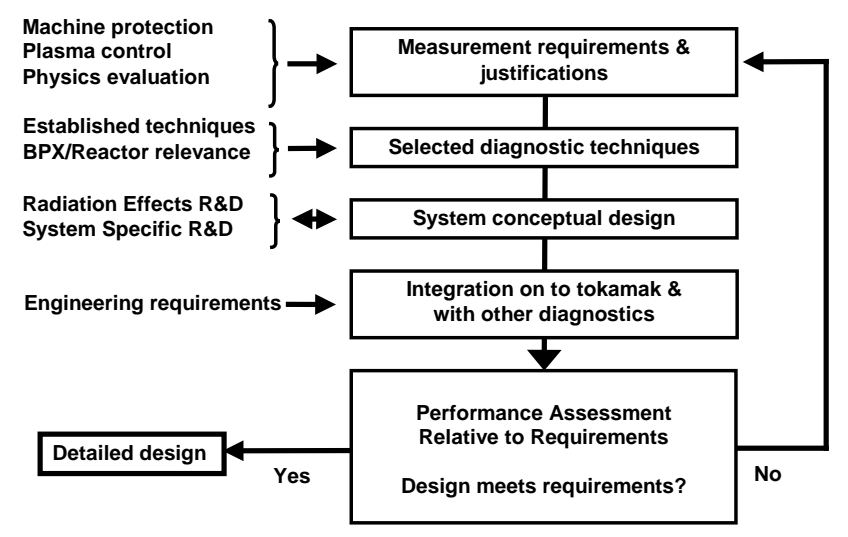

Figure 1: Simple flow diagram of the diagnostic design process. $^{2}$

\section{MEASUREMENT REQUIREMENTS}

The implementation of diagnostics on ITER must be carefully optimized. There is only limited space available for diagnostics and moreover, diagnostic systems add cost and complexity. Therefore priorities must be established with the obvious logic that systems that provide measurements for machine protection are given the highest priority, followed by those for basic plasma control and then advanced plasma control. In many cases performance evaluation and physics studies can be done with the data from the same systems, albeit that some times the diagnostic performance (resolution, number of channels) needs to be boosted. In some cases dedicated measurements are needed specifically for physics studies. These systems have the lowest priority on ITER. 
In order to meet all the measurement needs (machine protection, basic and advanced plasma control, physics evaluation) it is expected that about 45 - 50 different parameters will have to be measured. ${ }^{1,3}$ Table 1 in ref. [1] gives an overview of all measurements needed in various ITER operational scenarios ( $\mathrm{H}$ phase, $\mathrm{D}$ phase, $\mathrm{D} / \mathrm{T}$ phase, high power $\mathrm{D} / \mathrm{T}$ phase, hybrid and steady state operation) and also specifies which of these measurements are needed for control purposes.

For each of the parameters, detailed measurement requirements need to be developed that specify the spatial and temporal resolution and accuracy that are required for the measurement of the plasma parameter under various conditions. It is important in this process to aim for the minimum requirements that still support the measurement needs, and to not over specify (which would enhance cost and complexity). Therefore each of the measurement requirements needs to be accompanied by a detailed justification that states for what purposes specific accuracies, spatial and temporal resolutions are needed.

The development of the measurement requirements has been an on-going process since the start of the ITER Engineering Design Activity in 1992. ITER is the first magnetic confinement device for which the measurement requirements have been systematically developed during the machine design phase. This coherent approach has been taken in order to achieve an optimised, fully integrated, measurement system with a capability closely matched to the requirements. In case a specific requirement cannot be met for whatever reason, one can directly conclude from the justifications what part of the ITER research programme or machine operation will be affected. The development and fine-tuning of the measurement requirements (to keep track of developments in the field) has been, and still is, a substantial challenge on its own. A large fraction of the measurement requirements is based on experience at present devices and extrapolations towards ITER. However, ITER will be the first device operating in unexploited physics regimes with dominant heating by alpha particles. This implies that part of the measurement requirements had to be developed on the basis of modelling- where possible supported by experiments (e.g. alphaparticle simulation experiments ${ }^{4}$ ).

The full list of detailed measurement requirements is too long to be reproduced here. The reader is instead referred to Table 2 in ref. [1]. Since the publication of that table in 2007, some further changes have been made in the measurement requirements. The latest version of the table is contained in the ITER Project Integration Document, which is continuously updated, and can be found in the ITER Document Management System.

\section{SELECTION OF DIAGNOSTIC TECHNIQUES}

The second step in the design process (see Fig. 1) is to select the diagnostic techniques to perform all measurements.
Most techniques that are used in present devices can also be used on ITER, but their application is not always straightforward since the environment is much harsher. Additionally, many diagnostic functionalities are required that go the beyond the scope of those at present devices; e.g. in ITER even profiles of various parameters such as plasma rotation, electron temperature, current density are needed for control purposes of the more advanced plasma scenarios. So, it is important to carefully assess whether diagnostic techniques established at present fusion devices can be made more robust to operate in the harsh ITER environment and whether - if applicable - their scope can be expanded. Table 1 gives an overview of the diagnostic techniques that are presently foreseen for ITER.

\begin{tabular}{|c|c|}
\hline Magnetic Diagnostics & $\begin{array}{l}\text { Spectroscopic and NPA Sys- } \\
\text { tems }\end{array}$ \\
\hline $\begin{array}{l}\text { Vessel Magnetics } \\
\text { In-vessel magnetics } \\
\text { Divertor Coils } \\
\text { Continuous Rogowski Coils } \\
\text { Diamagnetic Loop } \\
\text { Halo Current Sensors }\end{array}$ & $\begin{array}{l}\text { CXRS Active Spectroscopy (+DNB) } \\
\text { H Alpha Spectroscopy } \\
\text { VUV Impurity Monitoring (Main } \\
\text { Plasma) } \\
\text { Visible \& UV Impurity Monitoring } \\
\text { (Divertor) }\end{array}$ \\
\hline Neutron Diagnostics & X-Ray Crystal Spectrometers \\
\hline $\begin{array}{l}\text { Radial Neutron Camera } \\
\text { Vertical Neutron Camera } \\
\text { Microfission chambers (in-vessel) } \\
\text { Neutron Flux Monitors (ex-vessel) } \\
\text { Gamma-Ray Spectrometers }\end{array}$ & $\begin{array}{l}\text { Visible Continuum Array } \\
\text { Soft X-Ray Array } \\
\text { Neutral Particle Analyzers } \\
\text { Laser Induced Fluorescence } \\
\text { MSE based on Heating Beam }\end{array}$ \\
\hline Neutron Activation Systems & Microwave Diagnostics \\
\hline $\begin{array}{l}\text { Lost Alpha Detectors } \\
\text { Knock-on Tail Neutron Spec- } \\
\text { trometer }\end{array}$ & $\begin{array}{l}\text { ECE Diagnostics for Main Plasma } \\
\text { Reflectometers for Main Plasma } \\
\text { Reflectometers for Plasma Position }\end{array}$ \\
\hline Optical/IR Systems & Reflectometers for Divertor Plasma \\
\hline $\begin{array}{l}\text { Thomson Scattering (Core) } \\
\text { Thomson Scattering (Edge) } \\
\text { Thomson Scattering (X-point) } \\
\text { Thomson Scattering (Divertor) }\end{array}$ & $\begin{array}{l}\text { ECA for Divertor Plasma } \\
\text { Microwave Scattering (Main } \\
\text { Plasma) } \\
\text { Fast Wave Reflectometry }\end{array}$ \\
\hline $\begin{array}{l}\text { Toroidal } \\
\text { interferometer/Polarimeter } \\
\text { Poloidal Polarimeter }\end{array}$ & $\begin{array}{l}\text { Plasma-Facing Component \& } \\
\text { Operational Diagnostics }\end{array}$ \\
\hline Collective Thomson scattering & IR Cameras, visible/IR TV \\
\hline Bolometric Systems & Thermocouples \\
\hline $\begin{array}{l}\text { Bolometric Array (Main Plasma) } \\
\text { Bolometric Array (Divertor) }\end{array}$ & $\begin{array}{l}\text { Pressure Gauges } \\
\text { Residual Gas Analyzers } \\
\text { IR Thermography Divertor } \\
\text { Langmuir Probes }\end{array}$ \\
\hline
\end{tabular}

Table 1: Overview of the diagnostics foreseen at ITER

ITER will be the first fusion device with dominant alpha heating. Because this is a new and unexploited regime, ${ }^{5}$ a range of new measurements is needed (e.g. the measurement of confined and escaping alpha particles, 
alpha-particle driven instabilities) for which novel techniques need to be developed. Whenever possible these should be tested on present devices. Specific examples of such diagnostics are fast ion collective Thomson scattering for studying fast particles including alphas, ${ }^{6}$ fast-wave reflectometry for measuring the fuel ion ratio, ${ }^{7}$ and techniques to measure escaping alpha particles. ${ }^{8}$

\section{ENVIRONMENTAL EFFECTS}

The third step in the diagnostics design process (see Fig. 1) is the system specific and radiation effects research and development. Diagnostics in ITER have to cope with an extremely harsh environment. ${ }^{1,9,10}$ Compared to the discharge of JET with the highest fusion performance thus far of $16 \mathrm{MW}$ during 1 second, ${ }^{4}$ ITER will have:

- neutral particle fluxes emerging from charge exchange reactions that are about a factor of 5 higher;

- neutron and gamma fluxes that are both an order of magnitude higher;

- plasma discharges that are about three orders of magnitude longer;

- neutron fluences that are at least $>10^{5}$ times higher;

- nuclear heating up to $1 \mathrm{MW} / \mathrm{m}^{3}$ (at JET essentially zero).

Especially the copious amounts of neutrons and gammas give rise to a range of phenomena that are new to the diagnostics and that should be incorporated in the system designs. Many of these phenomena deteriorate the proper functioning of electrical components:

- radiation-induced conductivity (RIC);

- radiation-induced electrical degradation (RIED);

- radiation-induced electromotive force (RIEMF);

- radiation-induced thermo-electric sensitivity (RITES);

- thermal-induced electromotive force (TIEMF).

Some of these effects depend on the irradiation dose whereas others depend on the dose-rate. Even straightforward components such as mineral insulated cables used to transmit signals from in-vessel detectors to data acquisition equipment outside the tokamak hall, and coils to measure magnetic fields in the vacuum vessel, suffer from these effects. The challenge is to develop cables, coils, diagnostic components, etc., in which the combined action of all effects does not deteriorate the proper functioning of the diagnostic. ject to:

Refractive materials (lenses, fibers, windows) are sub-

- radiation-induced absorption (RIA) and

- radio-luminescence or radiation-induced emission (RL or RIE; see Fig. 2).

Therefore, refractive materials can in general not be used at close proximity to the plasma, even though these effects can be reduced to some extend by hydrogen hardening and/or annealing at elevated temperatures (see Fig. 3). For most optical diagnostics this implies that it safe to use re- fractive components from the end of the port plug onwards. Inside the port plug only mirrors and/or waveguides can be used to transmit the radiation to and from the plasma.

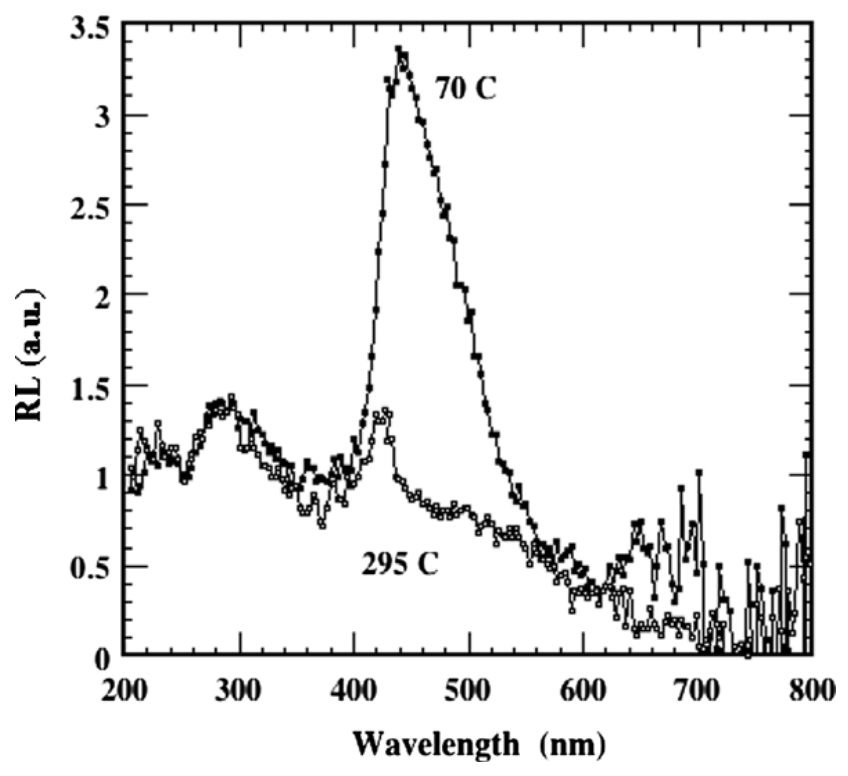

Figure 2: Low dose radio-luminescence ( $R L)$ spectra of $\mathrm{KS}-4 \mathrm{~V}$ (a Russian-made quartz fibre) at $700 \mathrm{~Gy} / \mathrm{s}$ and at temperatures of 70 and $295{ }^{\circ} \mathrm{C} .{ }^{11}$

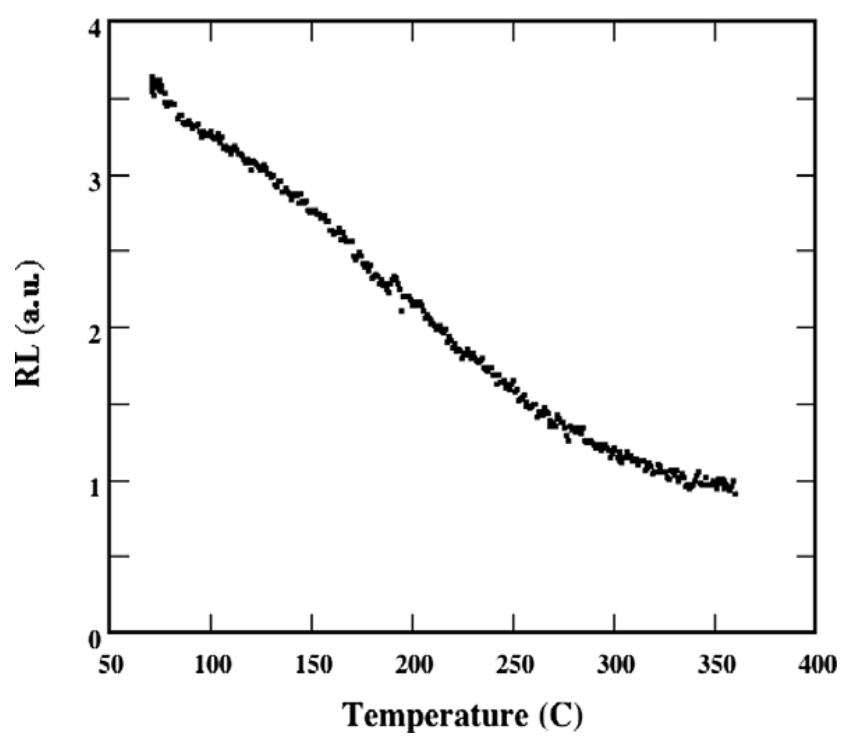

Figure 3: Thermal quenching of the $440 \mathrm{~nm}$ radioluminescence line at $700 \mathrm{~Gy} / \mathrm{s}^{11}$

Other effects caused by the high radiation levels are nuclear heating and changes in material properties such as swelling, transmutation ${ }^{12}$ and activation. All these effects can strongly affect the proper operation of diagnostics and diagnostic components. The challenge is to either use ma- 
terials that are robust to these effects or - in case this is impossible - to use alternative diagnostic techniques for measuring certain plasma parameters.

The nuclear environment sets also stringent demands on the engineering of the diagnostic systems. ${ }^{9}$ The diagnostics should be designed in such a way that neutrons are prevented from streaming out of the vessel. For many optical and microwave diagnostics this can be achieved by avoiding straight viewing lines; instead the viewing lines can be folded by using mirrors or waveguide bends, respectively. All diagnostics should have a double tritium barrier with a monitored volume in between (see Fig. 4). A further complication is that all components inside the vacuum vessel and inside the port plugs must be capable of being repaired or replaced with remote handling tools. (see Fig. 5).

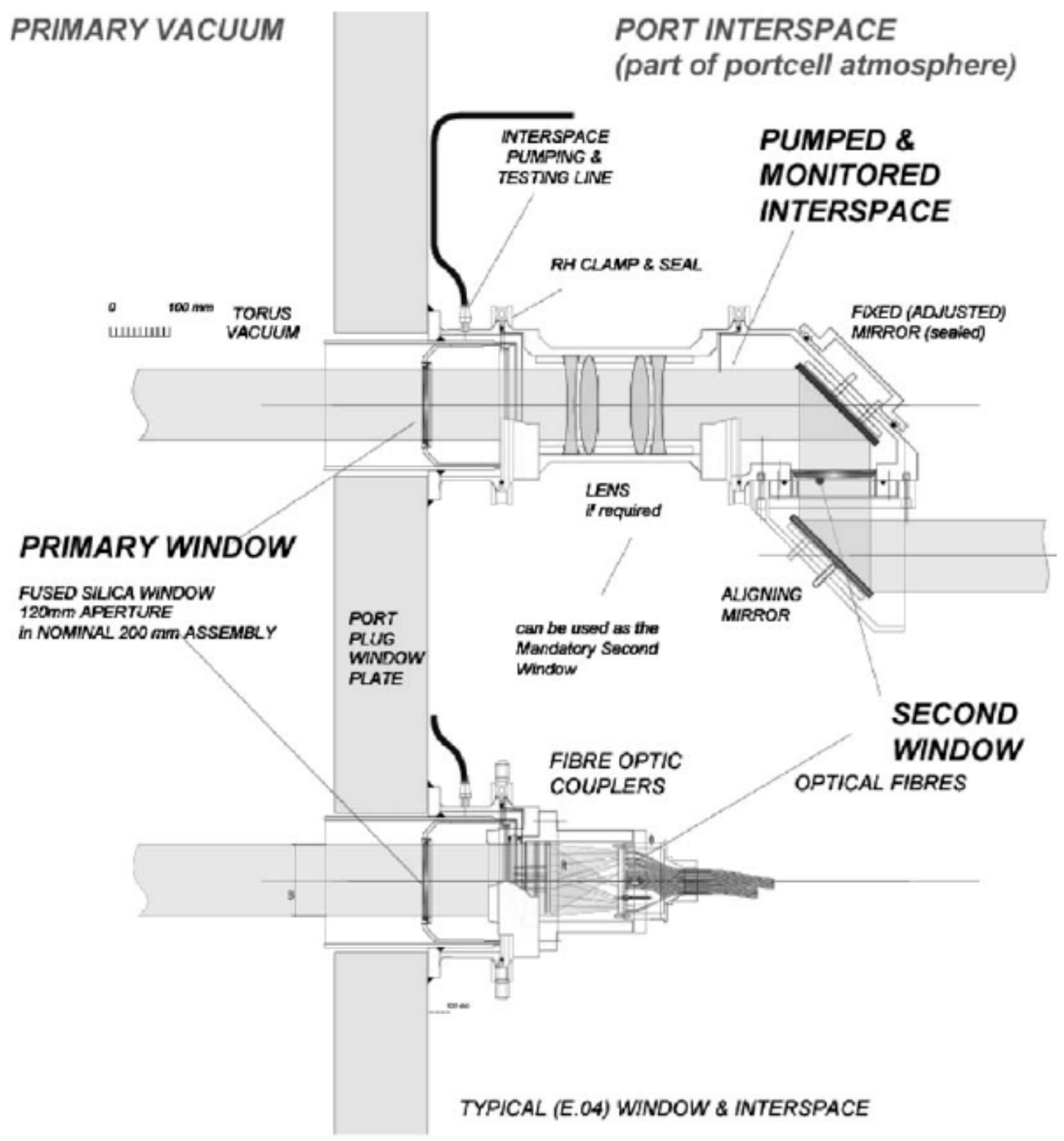

Figure 4: Typical example of two diagnostic window arrangements in ITER. ${ }^{9}$ 


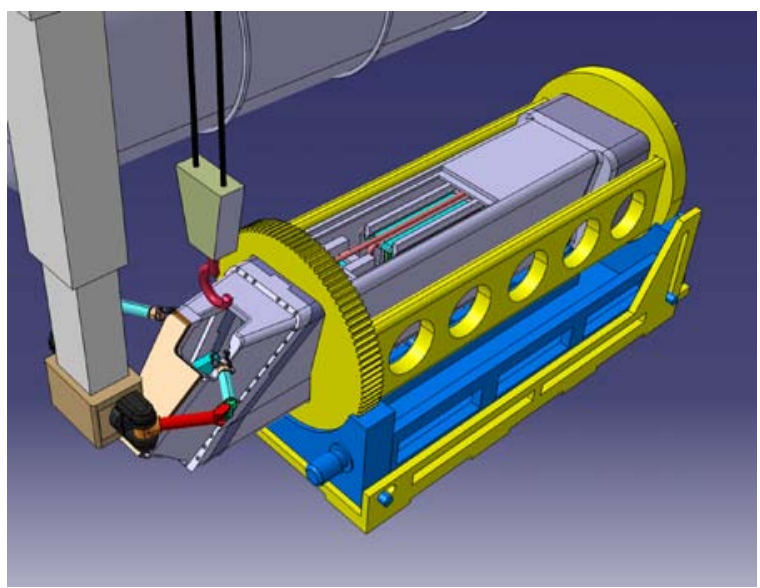

Figure 5: Remote Handling Operation on one of the ITER Port Plugs in the Hot Cell. Courtesy B.S.Q. Elzendoorn.

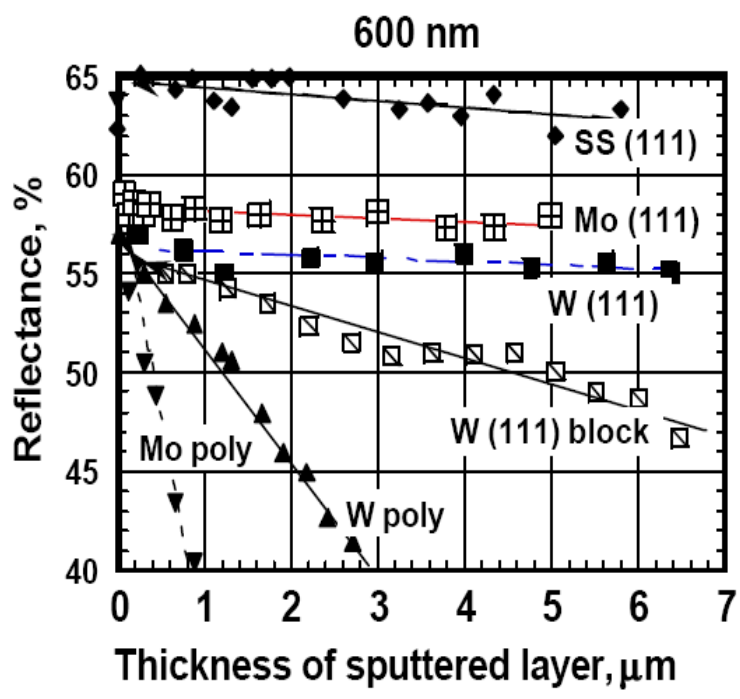

Figure 6: Reflectance of stainless steel (SS), $W$ and Mo mirrors of different structures (polycrystalline, block single crystalline and single crystalline with (111) planes of orientation) at $600 \mathrm{~nm}$ depending on the thickness of the layer eroded due to bombardment by ions of deuterium plasma. (Adapted from [13]).

So mirrors seem to the 'magic' solution. At the one hand they can be applied instead of refractive components that suffer from RIA and RIE, while at the other hand they make it possible to fold the optics path into a labyrinth to suppress the neutron streaming. However, nothing comes for free: The high neutral particle fluxes emerging from charge exchange reactions lead to erosion and redeposition elsewhere. Both of these effects can strongly affect the polarization dependent reflectivity of the mirrors, and thereby deteriorate the proper functioning of the diagnostics. ${ }^{14}$ Erosion is the least of the two problems, since in erosion dominated regions it is possible to use single crystalline mirrors of materials like tungsten, molybdenum, and stainless steel that are rather resistant to erosion. Moreover, the reflectivity of these single crystalline mirrors does not strongly depend on the thickness of the eroded layer (see Fig. 6).

In contrast, deposition has in almost all cases a strong deleterious effect on the reflective properties of the mirrors (see Fig. 7). Much work is done in order to find the optimum mirror materials and also the best geometries for especially the first mirror, which is least vulnerable to erosion and especially deposition. Furthermore, attention is devoted to develop means for mirror protection (e.g. shutters, gas flow in front of the mirrors) or to mitigate deposition (e.g. operating the mirrors at elevated temperatures). In DIII-D it has been demonstrated that mirrors in the divertor heated to $200-300{ }^{\circ} \mathrm{C}$ suffer much less from deposits than mirrors at the same place, operated at room temperature (see Fig. 8). ${ }^{15}$

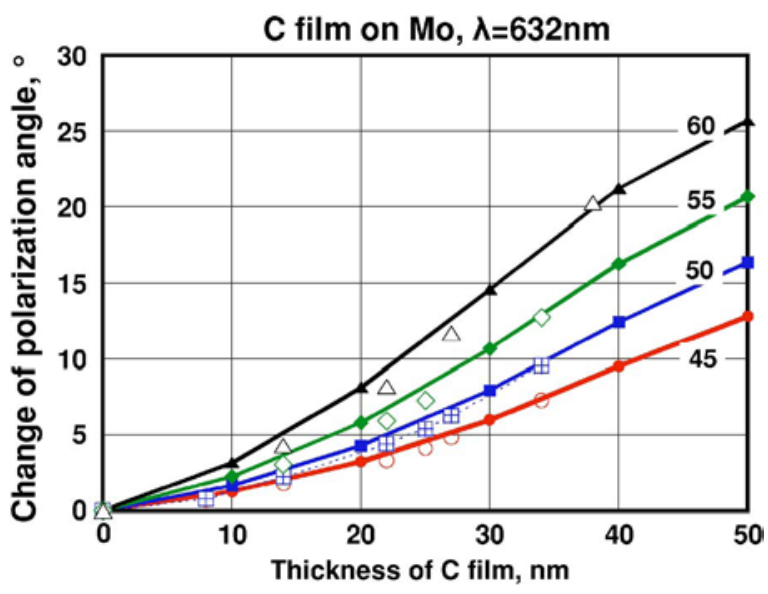

Figure 7: Calculated (lines and solid points) and experimental values (open points) for the change of the polarization angle of light at $632 \mathrm{~nm}$ reflecting from a Mo mirror coated with different thicknesses of $C$ film. The angle of incidence is shown near every curve. $^{16}$

Another diagnostic challenge arises from the long duration of the ITER discharges. These gives rise to problems for traditional techniques such as inductive magnetic pick-up coils for measuring magnetic fields. Especially the integrators are vulnerable to drift. The combination of the long pulse and the high levels of radiation constitute a rather difficult measurement problem. In addition to trying to make these techniques more robust and radiation hard, research is on-going to develop radiation-hard steady state magnetic field sensors based on the Hall effect, ${ }^{17}$ and to develop alternative techniques such as position reflectometry ${ }^{18}$ to measure the gap between the density at the separatrix and the first wall.

A final challenge for diagnostics that will be mentioned in this paper is caused by the high temperatures in ITER, which can reach values up to about $40 \mathrm{keV}$. This gives rise to strong relativistic effects, which have a large effect on microwave diagnostics because of the downshifted electron cyclotron emission (ECE). The result is that in contrast to 
present devices, ECE using the $2^{\text {nd }}$ harmonic X-mode cannot be used for determining the electron temperature $\mathrm{T}_{\mathrm{e}}$ in the plasma core, due to overlap by relativistically downshifted $3^{\text {rd }}$ harmonic emission (see Fig. 9). Instead the $1^{\text {st }}$ harmonic O-mode needs to be utilized. The downshifted emission also strongly limits the operational ranges of reflectometers to measure the density profile. The core density profile can only be measured by reflectometry via the lower cutoff from the high field side. Reflectometry on the plasma frequency from the low field side only can yield the density profile in the vicinity of the separatrix.

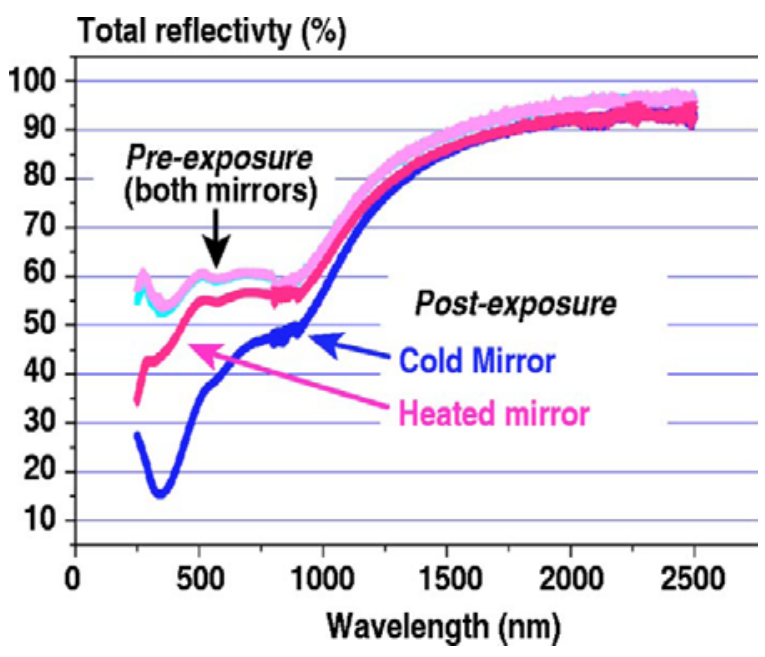

Figure 8: Total reflectivity of heated and non-heated mirrors before and after exposures in the DIII-D divertor. ${ }^{15}$

\section{INTEGRATION OF DIAGNOSTICS}

The fourth step in the diagnostic design process (see Fig. 1) is the engineering design and integration of all diagnostics on the tokamak. ITER will have five diagnostic ports at the equatorial level and twelve at the upper level. Additionally, a number of special instrumented cassettes are foreseen in the divertor. In contrast to present-day machines where each diagnostic is implemented on its own diagnostic port, there is a need to install several diagnostics in a single diagnostic port (see Fig. 10). ${ }^{9,19}$ This puts of course high demands on the integration of all systems. In most cases the various diagnostics will be developed by different teams in the ITER parties. Effects such as the neutron shielding, the total mechanical strength of the port plug and deformations under ITER operation have to be assessed for the full port plug, and these assessments must be redone if changes are made to the design of the individual diagnostics. Due to the multitude of interfaces between the various diagnostics and between the parties working on them, this is far from being an easy task.

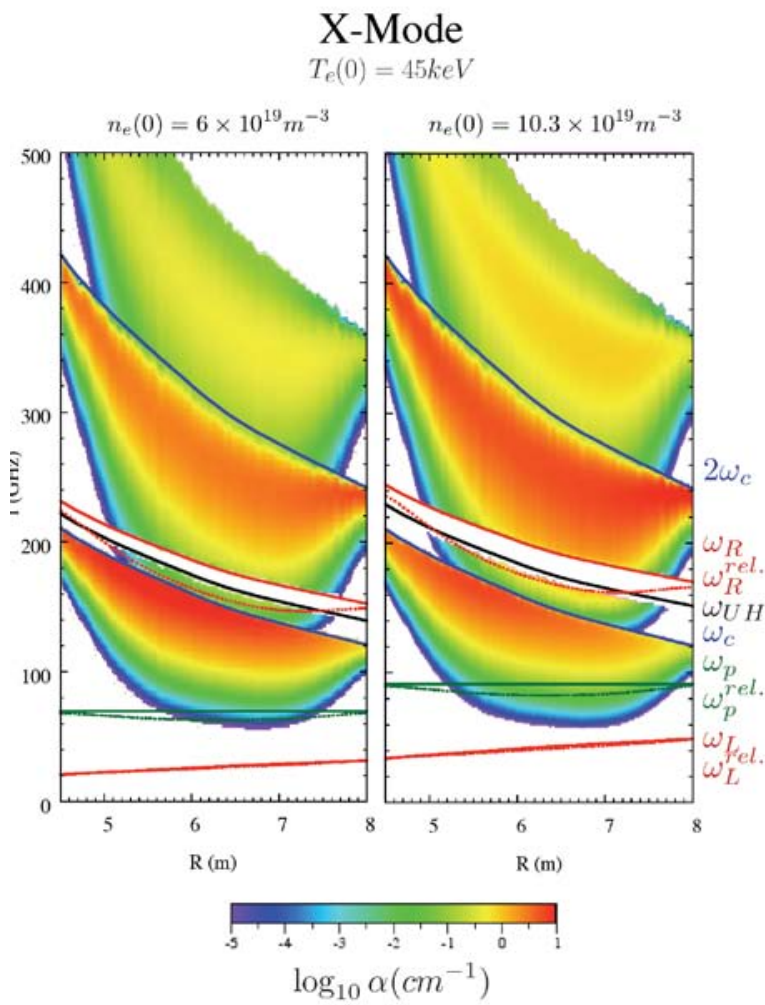

Figure 9: Local absorption contours for $X$-mode propagation at perpendicular incidence for two densities, at high temperature ( $T_{e}$ on axis is $45 \mathrm{keV}$ ) representing an extreme case of what might occur in an

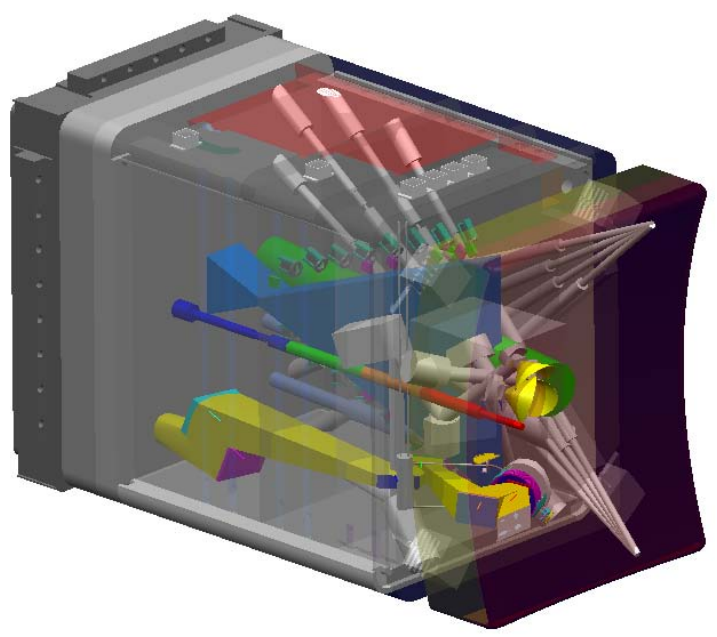

ITER steady-state scenario Adapted from [20].

Figure 10: Cut-away view of equatorial port number 1 providing access for the radial neutron camera, high resolution neutron spectrometer, a visible IR-TV camera, gamma ray spectrometers, bolometry, one of the divertor impurity monitors, and one of the viewing systems for Motional Stark Effect diagnostic (courtesy C.I. Walker, ITER IO).

\section{DIAGNOSTIC ASSESSMENT}

The final step in the diagnostic design process (see Fig. 1) is the assessment of the diagnostic capability with respect to the measurement requirements. This 
is done rather frequently. Although there have been more recent assessments, Table 7 in ref. [1] is still actual. For roughly $50 \%$ of the plasma parameters it is expected that the measurement requirements can be met, for about the same number of other plasma parameters it is expected that the measurement requirements can be partly met. For three parameters it is expected that the measurement requirements can not be met. These are the measurements of alpha particle losses (escaping alpha particles), the ion temperature in the divertor and the plasma flow in the divertor. For these parameters it is necessary to go back to the measurement requirements (see Fig. 1) to judge whether they can be somewhat relaxed without harming the operation of the machine or the intended physics programme. If this is not possible it is necessary to try to improve the diagnostic design and/or to develop novel techniques in order to meet the requirements.

\section{DIAGNOSTIC FOR NEXT STEP DEVICES}

The diagnostic design process for next step devices as $\mathrm{DEMO}^{21}$ will be in principle similar to the one sketched in Fig. 1. But DEMO will have higher levels of neutron flux, fluence, nuclear heating, gamma irradiation and plasma irradiation than ITER. In particular the neutron fluence will be $\sim 50$ times higher than in ITER, while the neutron flux is a few times higher. This implies that techniques or diagnostic components that marginally work in the ITER environment are likely to be inappropriate in DEMO. This applies to many in-vessel diagnostic components (e.g. cables, magnetic coils and bolometry), for which there seems little prospect for improvements that would make it possible to use them in DEMO in a similar way as in ITER. ${ }^{22,23}$

The particle flux in DEMO is expected to be $\sim 2$ times higher with respect to ITER. Even this 'small' increase may make optical systems with largeaperture mirrors problematic, given the very difficult situation already in ITER. Small apertures and mirrors recessed far into the shielding, and use of exchangeable optical fibres, could make optical diagnosis possible, but with very limited views of the DEMO plasma. Techniques that still seem feasible without large modifications from the present practice are microwave techniques and direct line-of-sight techniques (e.g. neutrons and x-rays). As mainly the neutron fluence (and not the flux) in DEMO is larger than in ITER, i.e. effects in relation to lifetime, there may be prospects for use of in-vessel diagnostic components such as magnetic coils and bolometers if provisions are made that they can be replaced regularly. The same is true for larger mirrors. If such invessel diagnostics and optical diagnostics with largeaperture mirrors are deemed essential for DEMO, schemes must be enabled for regular replacement, which may significantly impact on the DEMO design.
These considerations lead to the following needs for R\&D for DEMO diagnostics. New diagnostic techniques need to be developed that are suitable for the DEMO environment, in particular to replace the established diagnostic techniques that will be problematic in DEMO. For in-vessel components, there may be little prospect other than ensuring they can be replaced frequently as discussed above. For ex-vessel components the situation may be less critical. Nevertheless, this is an area that needs intensive study, requiring testing and qualification on fission reactors, ITER (full-power DT phase) and the International Fusion Materials Irradiation Facility. ${ }^{24}$ It should be noted that irradiation testing, even at ITER-relevant levels, is time consuming and costly. The fissionreactor time needed to reach DEMO-relevant fluences may therefore be problematic. Work on ITER needs to guide the selection of techniques that can best cope with the harsh environment. During the ITER life time, experience should be gained with real-time data handling and validation to process large quantities of data, in-pulse calibration, etc.

ITER will routinely have DT plasmas and the alpha heating effect will be significant. Alpha particle physics and alpha induced instabilities can therefore be studied in full detail and the impact of those on DEMO can be assessed (along with a selection of the optimum diagnostics techniques).

Unlike ITER, DEMO will not be designed as a flexible research tool, since it needs to demonstrate the economics of the fusion reactor. It is expected that DEMO will have only 1 - 2 different (advanced) plasma operating scenarios. The selection of these scenarios needs to be done during the full power DT phase in ITER. The limited number of operating scenarios implies that likely a smaller number of measurements/diagnostics are required. Once the DEMO scenarios have been selected in ITER, a high number of essentially identical high performance pulses are needed in order to determine the minimum set of diagnostics that fully supports the DEMO scenarios. One should realize, however, that the lack of suitable diagnostic techniques may affect the options for controlling the DEMO plasma, in particular the ability to run "advanced" scenarios. This lack of diagnostics, and thus the reduced set of plasma parameters that can be measured, will make modelling more important in DEMO to derive the other parameters. Advanced predictive/analysis codes need to be developed to combine data from various diagnostics in an intelligent way in order to reduce the number of required diagnostics. A successful strategy is to use socalled "dynamic state observers", which is in essence a real-time simulation of a theoretical model of the plasma, ${ }^{25}$ running parallel to the physical evolution of the plasma in the tokamak. The model predictions are continuously compared to the available diagnostic measurements, yielding improved estimates and/or leading to slight adaptations in the model. The actual control then uses the state estimate from the 
observer, on a timescale independent from (and often faster than) the diagnostic measurements.

\section{ACKNOWLEDGMENT}

This paper is largely based on the experience of the author as chair of the ITER Expert Group on Diagnostics, the ITPA and the EFDA Topical Group on Diagnostics. He would like to thank all colleagues that have contributed to the work of these groups thus forming the basis of this paper. The paper is a slightly revised version of the paper that has been written for the 2009 and 2011 Carolus Magnus Summer Schools. The author is indebted to Ben Elzendoorn, Eric Hodgson, Dmitry Rudakov, George Vayakis and Chris Walker for giving permission to use their figures. This work, supported by the European Communities under the contract of Association between EURATOM/FOM, was carried out within the framework of the European Fusion Programme with financial support from NWO and ITER-NL. The views and opinions expressed herein do not necessarily reflect those of the European Commission.

\section{REFERENCES}

1 A.J.H. DONNÉ, et al., "Progress in ITER Physics Basis, Chapter 7 : Diagnostics”, Nucl. Fusion 47, S337 (2007)

2 A.J.H. DONNÉ and A.E. COSTLEY, “Key Issues in Diagnostics for Burning Plasma Experiments”, IEEE Transact. Plasma Sci. 32 (2004) 177.

3 K. EBISAWA et al., "Plasma Diagnostics for ITER-FEAT”, Rev. Sci. Instrum. 72, 545 (2001).

4 P.R. THOMAS et al., "Overview of Alpha Heating in JET DT Plasmas”, Phys. Rev. Lett. 80, 5548 (1998).

5 S.E. SHARAPOV et al., "Burning Plasma Studies at JET”, Fusion Sci. Techn. 53, 989 (2008).

6 E. TSAKADZE et al., "Fast Ion Collective Thomson Scattering for ITER: Design Elements”, Fusion Sci. Techn. 53, 69 (2008).

7 L. CUPIDO et al., "High Resolution Fast Wave Reflectometry: JET Design and Implications for ITER”, Rev. Sci. Instrum. 79, 10F106 (2008).

8 M. SASAO et al., "Fusion Product Diagnostics", Fusion Sci. Techn. 53, 604 (2008).

9 G. VAYAKIS et al., "Generic Diagnostic Issues for a Burning Plasma”, Fus. Sci. Techn. 53, 699 (2008).

10 A.E. COSTLEY et al., "ITER R\&D: Auxiliary Systems: Plasma Diagnostics”, Fusion Eng. Design 55, 331 (2001).
11 A. MOROÑO and E.R. HODGSON, "Radioluminescence Behaviour for Electron Irradiated KS-4V”, J. Nucl. Mat. 367-370, 1107 (2007).

12 M. GONZALEZ and E.R. HODGSON, "Radiation Resistant Bolometers using Platinum on $\mathrm{Al}_{2} \mathrm{O}_{3}$ and AIN”, Fusion Eng. Design 74, 875 (2005).

13 A.E. COSTLEY et al., "ITER R\&D: Auxiliary Systems: Plasma Diagnostics”, Fusion Eng. Design 55, 331 (2001).

14 A. LITNOVSKY et al., "Progress in Research and Development of Mirrors for ITER Diagnostics”, Nucl. Fusion 49, 075014 (2009).

15 D. RUDAKOV et al., "First Tests of Molybdenum Mirrors for ITER Diagnostics in DIII-D Divertor”, Rev. Sci. Instrum. 77, 10F126 (2006).

16 V. VOITSENYA et al., "Simulation of Environmental Effects on Retroreflectors in ITER", Rev. Sci. Instrum. 76, 083502 (2005).

17 I. BOLSHAKOVA et al, "Performance of Hall Sensor-based Devices for Magnetic Field Diagnosis at Fusion Reactors", Sensor Lett. 5, 283 (2007).

18 F. DA SILVA, et al., "Developments of Reflectometry Simulations for Fusion Plasmas: Application to ITER Position Reflectometers”, J. Plasma Phys. 72, 1205 (2006).

19 C.I. WALKER et al., "ITER Diagnostics: Integration and Engineering Aspects”, Rev. Sci. Instrum. 75, 4243 (2004).

20 G. VAYAKIS et al., "Status and Prospects for mm-Wave Reflectometry in ITER", Nucl. Fusion 46, S386 (2006).

21 D. MAISONNIER, "European DEMO Design and Maintenance Strategy", Fusion Eng. Design 83, 858 (2008).

22 A.J.H. DONNÉ et al., "Diagnostics for plasma control on DEMO: challenges of implementtation”, Nucl. Fusion 52, 074015 (2012).

23 A.J.H. DONNÉ, "From present Fusion Devices to DEMO: a changing Role between Diagnostics and Modeling”, accepted for publication in Plasma Fusion Res. 8, 2102084 (2013).

24 P. GARIN and M. SUGIMOTO, "Main Baseline of IFMIF/EVEDA Project", Fusion Eng. Design 84, 259 (2009).

25 F. FELICI et al., "Real-time physics-model-based simulation of the current density profile in tokamak plasmas ”, Nucl. Fusion 51, 083052 (2011). 